Biochemical Engineering Journal

Elsevier Editorial system(tm) for

Manuscript Draft

Manuscript Number: BEJ-D-16-00782R1

Title: Engineering Yarrowia lipolytica for efficient $\gamma$-linolenic acid production

Article Type: Full Length Article

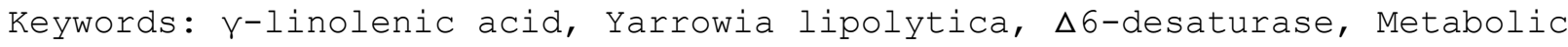
engineering, Temperature-shift strategy

Corresponding Author: Dr. Xiao-Jun Ji, Ph.D.

Corresponding Author's Institution: Nanjing Tech University

First Author: Mei-Li Sun

Order of Authors: Mei-Li Sun; Catherine Madzak; Hu-Hu Liu; Ping Song; LuJing Ren; He Huang; Xiao-Jun Ji, Ph.D.

Abstract: $Y$-linolenic acid (GLA) has various well-documented beneficial physiological effects and high biological significance. Because the natural supply of GLA is insufficient, microbial GLA production is a promising method for pharmaceutical and nutraceutical purposes. To establish and develop a biotechnological process for GLA production by Yarrowia lipolytica, the codon-optimized $\Delta 6$-desaturase from Mortierella alpina was introduced into this yeast under the control of the strong hp4d promoter. A recombinant $Y$. lipolytica strain was constructed, which produced $4.6 \%$ GLA in total fatty acids. By using a temperature-shift strategy of cultivation, consisting in preliminary growth at $28{ }^{\circ} \mathrm{C}$ followed by 6 -day culture at $20^{\circ} \mathrm{C}$, optimal levels of dry cell weight (DCW), lipid content and GLA concentration were obtained from the recombinant strain: $18.55 \mathrm{~g} / \mathrm{L}, 1.16 \mathrm{~g} / \mathrm{L}$ and $71.6 \mathrm{mg} / \mathrm{L}$, respectively. These DCW, lipid and GLA values were respectively $25.7 \%$, 19.6\% and 60.9\% higher than those obtained in the control cultivation experiment at the standard constant temperature of $28{ }^{\circ} \mathrm{C}$. This work demonstrates the excellent capacity of Y. lipolytica for GLA production, by combining metabolic engineering with a temperature-shift strategy. 
Dear Editors,

On behalf of our co-authors, we are submitting the enclosed manuscript entitled 'Engineering Yarrowia lipolytica for efficient $\gamma$-linolenic acid production' for possible publication in 'Biochemical Engineering Journal'. The classification for this manuscript should be: '120.040: metabolic engineering'.

We believe that several aspects of this paper may be of particular interests to the readers of your journal. Chiefly, the codon-optimized $\Delta 6$-desaturase from Mortierella alpina was successfully introduced in Yarrowia lipolytica Polf and the effect of $\Delta 6$-desaturase on cell growth, lipid accumulation, and fatty acid synthesis were systematically examined. Then using a temperature-shift strategy of cultivation, dry cell weight (DCW), lipid content and GLA concentration was improved significantly.

Firstly, the codon-optimized $\Delta 6$-desaturase was expressed in $Y$. lipolytica using p0 vector driven by hp4d promoter. To investigate the discrepancy between the control strain and the genetically modified strain, the fermentation performance of the two strains was comparable. It was observed that GLA represented $4.6 \%$ of total fatty acids in the engineered strain, when it was not detected in the control strain Po1f. In addition, the introduction of $\Delta 6$-desaturase improved the value of DCW and increased the glucose consumption rate, but didn't affect lipid yield. Then, fermentation conditions were modified using a temperature-shift strategy. The engineered strain was consequently cultivated at $15{ }^{\circ} \mathrm{C}, 20{ }^{\circ} \mathrm{C}$ and $28{ }^{\circ} \mathrm{C}$. Based on the fermentation results at different temperature, a controlled temperature-shift processes were developed: consisting in preliminary growth at $28^{\circ} \mathrm{C}$ followed by 6 -day culture at $20{ }^{\circ} \mathrm{C}$, optimal levels of dry cell weight (DCW), lipid content and GLA concentration were obtained from the recombinant strain were respectively $25.7 \%, 19.6 \%$ and $60.9 \%$ higher than those obtained in the control cultivation experiment of $28{ }^{\circ} \mathrm{C}$. The two-stage culture strategy proposed here, based on $Y$. lipolytica fermentation characteristics, could be applied to improving production of many other metabolites.

We certify that we have participated sufficiently in the work to take public responsibility for the appropriateness of the experimental design and method, and the collection, analysis, and interpretation of the data. We have reviewed the final version of the manuscript and approve it for publication. To the best of our knowledge and belief, this manuscript has not been published in whole or in part nor is it being considered for publication elsewhere. Also, the manuscript was not previously submitted to Biochemical Engineering Journal.

Please feel free to contact us if you have any questions or need additional information from us concerning this submission.

Sincerely yours,

Xiao-Jun Ji, Ph.D., Associate Professor

Tel: +86 25 58139342; Fax: +86 2558139389

E-mail: xiaojunji@njtech.edu.cn 


\section{Detailed Response to Reviewers}

Manuscript Number: BEJ-D-16-00782R1

Manuscript Title: Engineering Yarrowia lipolytica for efficient $\boldsymbol{\gamma}$-linolenic production

Article Type: Full Length Article

Dear Editor,

We would like to thank the anonymous referees for their kind comments and valuable suggestions. We are pleased to answer the questions of the reviewers' and the manuscript (Manuscript number: BEJ-D-16-00782R1) has also been extensively revised according to the comments (resubmitted online).

The answers also have been summarized one by one as listed below.

Finally, we sincerely hope that the revised manuscript can meet the standard for publication in your journal.

PS: The modifications were marked in red in the revised manuscript.

Thank you very much.

Sincerely yours,

\section{Xiao-Jun Ji}

\section{Reviewer comments:}

Reviewer \#1:

This manuscript by Sun et al. describes a two-prong approach to engineering a $Y$. lipolytic to produce gamma-linolenic acid (GLA). Specifically, the authors integrate a heterologous delta6-destaturase gene to convert linolenic acid to gamma-linolenic acid and use a two-stage variable temperature bioprocess to increase production. The manuscript is clear, well-written and the conclusions are justified. While the achieved titers of GLA are not overly exciting, this work advances the understanding of the process and provides new data on two-stage bioprocesses with a low-temperature production phase. Please consider the following minor points:

Answer: Thank you for your recognition of our work. Your suggestion is very pertinent and helpful for our work. And we have revised the manuscript carefully one by one according to your suggestion as follows.

\section{Minor comments:}

1. The genome editing tools for $Y$. lipolytica have recently been expanded to include CRISPR-Cas 9 systems. Please add current references to line 49 of page 3 (e.g., DOI: 10.1021/acssynbio.5b00162)

Answer: Thank you for your reminder. The current references below were added to line 49 of page 3 and the reference list. We have also changed the reference number in 
the reference list accordingly (marked in red).

C.M. Schwartz, M.S. Hussain, M. Blenner, I. Wheeldon, Synthetic RNA polymerase III promoters facilitate high-efficiency CRISPR-Cas9-mediated genome editing in Yarrowia lipolytica, ACS. Synth. Biol. 5 (2016) 356-359.

S. Gao, Y. Tong, Z. Wen, L. Zhu, M. Ge, D. Chen, Y. Jiang, S Yang, Multiplex gene editing of the Yarrowia lipolytica genome using the CRISPR-Cas9 system, J. Ind. Microbiol. Biot 43 (2016) 1085-1093.

2. Please provide a reference to the hp4d promoter and its quasi-constitutive function. Answer: Thank you for your reminder. We have added the reference in line 31 of page 9 (marked in red).

C. Madzak, B. Tréton, S. Blanchin-Roland, Strong hybrid promoters and integrative expression/secretion vectors for quasi-constitutive expression of heterologous proteins in the yeast Yarrowia lipolytica, J. Mol. Microbiol. Biotechnol. 2 (2000) 207-216.

3. Please expand the discussion of the increase in GLA production at low temperature. For example, what is the reasoning behind the increase in ricinoleic acid (ref 33) at low temperature, does the same reasoning apply here?

Answer: Thank you for your suggestion. I am sorry for my unclear expression before. In the revised manuscript, we have expanded the discussion of the increase in GLA production when shifted to lower temperature to line 37 of page 18 (marked in red).

"Zheng et al. [36] demonstrated specific cell growth rate are higher in the log phase than that of in the stationary phase. Thus, the cells shifted to lower temperature in the $\log$ phase were competent to produce a high level of GLA as described in the work of R. Holic et al. [35]."

The references were also added in the reference list.

M. Zheng, G.C Du, W.F. Guo, J. Chen, A temperature-shift strategy in batch microbial transglutaminase fermentation, Process Biochem. 36 (2001):525-530.

\section{Reviewer \#2:}

This manuscript describes the production of GLA in $Y$. lipolytica by expression a Delta- 6 desaturase, building on previous work from the authors. The main contribution of the present paper was to show that temperature shifts increase the production of GLA. Overall, I think this is a very interesting results. I never thought of temperature before as tuning variable -- in these regards, the results are novel. I recommend publication with a few minor suggestions for improving the manuscript. 
Answer: Thank you for your recognition of our work. Your suggestion is very pertinent and helpful for our work. And we have revised the manuscript carefully one by one according to your suggestion as follows.

\section{Minor comments:}

1. Please provide the codon optimization table and the sequence for the Delta-6 desaturase. This is necessary so that others can reproduce the results. I did not see this unless I am missing something.

Answer: Thank you for your suggestion. We have provided the sequence for codon-optimized $\Delta 6$-fatty acid desaturase and $\Delta 6$-fatty acid desaturase in Supplementary Table S1.

\section{Supplementary Table 1}

The sequence for codon-optimized $\Delta 6$-fatty acid desaturase and $\Delta 6$-fatty acid desaturase

\begin{tabular}{|c|c|}
\hline Gene & Sequence \\
\hline \multirow{27}{*}{$\begin{array}{l}\text { codon- } \\
\text { optimized } \\
\Delta 6 \text {-fatty } \\
\text { acid } \\
\text { desaturase }\end{array}$} & ATGGCCGCCGCCCCCTCCGTCCGAACCTTCACCAGAGCCG \\
\hline & AAATCCTGAACGCTGAGGCCCTGAACGAGGGCAAGAAGG \\
\hline & ACGCCGAGGCCCCTTTCCTCATGATCATCGACAACAAGGT \\
\hline & GTACGACGTGCGAGAATTCGTGCCCGACCACCCTGGTGGT \\
\hline & TCCGTGATTCTGACCCATGTCGGTAAGGACGGCACTGACG \\
\hline & TCTTTGACACCTTCCACCCCGAGGCCGCTTGGGAAACCCT \\
\hline & GGCTAATTTTTATGTTGGTGACATTCACGAGTCTGACCGA \\
\hline & GACATCAAGAACGACGACTTTGCTGCCGAAGTCCGAAAG \\
\hline & CTGCGAACCCTGTTTCAGTCCCTGGGTTACTACGATTCTTC \\
\hline & TAAGGCCTACTACGCCTTCAAGGTCTCCTTCAACCTGTGC \\
\hline & ATCTGGGGCCTGTCCACCTTCGTCGTCGCCAAGTGGGGTC \\
\hline & AAACCTCTACCCTCGCTAACGTTGTTTCTGCTGCTCTGCTG \\
\hline & GGTCTGTTCTGGCAGCAGTGCGGTTGGCTCGCCCACGATT \\
\hline & TCCTGCACCATCAGGTCTTCCAGGACCGATTCTGGGGCGA \\
\hline & ССTCTTTGGCGCTTTCCTCGGTGGCGTGTGTCAGGGTTTCT \\
\hline & ССTCCTCCTGGTGGAAGGACAAGCACAATACCCACCACGC \\
\hline & CGCCCCCAACGTGCATGGTGAGGACCCCGACATCGACACT \\
\hline & САCССССТCСТGACCTGGTCCGAGCACGCTCTGGAGATGT \\
\hline & TCTCCGATGTCCCCGACGAGGAGCTCACCAGAATGTGGTC \\
\hline & CAGATTCATGGTGCTGAACCAGACCTGGTTCTACTTCCCC \\
\hline & ATCCTGTCTTTCGCCCGACTCTCCTGGTGCCTCCAGTCCAT \\
\hline & CCTCTTCGTCATGCCCAACGGTCAGGCTCATAAACCTTCT \\
\hline & GGTGCCCGAGTGCCTATTTCCCTGGTGGAACAGCTGTCCC \\
\hline & TCGCCATGCACTGGACCTGGTATTTAGCCACCATGTTCCT \\
\hline & GTTCGTGAAGGACCCTATCAACATGTTCGTGTACTTCCTG \\
\hline & GTGTCTCAGGCCGTGTGTGGCAACCTGCTGGCTCTGGTCT \\
\hline & TTTCTCTGAACCACAACGGCATGCCCGTCATCTCCAAGGA \\
\hline
\end{tabular}




\begin{tabular}{|c|c|}
\hline & $\begin{array}{l}\text { GGAGGCCGTCGACATGGATTTCTTCACCAAGCAGATCATC } \\
\text { ACCGGCCGAGATGTCCACCCTGGCCTGTTCGCCAACTGGT } \\
\text { TCACCGGCGGCCTGAACTACCAGATCGAGCATCACCTGTT } \\
\text { CCCCTCCATGCCCCGACACAACTTCTCCAAAATCCAGCCC } \\
\text { GCCGTGGAGACCCTCTGTAAGAAGTACAACGTGCGATACC } \\
\text { ACACTACCGGTATGATCGAGGGCACCGCCGAGGTCTTCTC } \\
\text { CCGACTGAACGAGGTCTCTCGAGCCGCCTCTAAGATGGGT } \\
\text { AAGGCTCAGTAA }\end{array}$ \\
\hline $\begin{array}{l}\Delta 6 \text {-fatty } \\
\text { acid } \\
\text { desaturase }\end{array}$ & $\begin{array}{l}\text { ATGGCTGCTGCTCCCAGTGTGAGGACGTTTACTCGGGCCG } \\
\text { AGATTCTGAATGCCGAGGCCCTGAATAAGGCAAGAAGG } \\
\text { ATGCTGAGGCACCCTTCCTGATGATCATCGACAACAAGGT } \\
\text { GTACGATGTCCGCGAGTTTTCCCTGATCATCCCGGGGA } \\
\text { AGTGTGATTCTCACGCACGTTGGCAAGGACGGCACTGACG } \\
\text { TCTTTGACACTTCCACCCCAGGCTGCTTGGGAGACTCTT } \\
\text { GCCAACTTTTAGTTGGTGATATTCATGAGAGCGACCGCG } \\
\text { ACATCAAGAATGATGACTTTGGGCCGAGGTTCGCAAGCT } \\
\text { GCGTACCTTGTTCAGTCTCTTGGCTACTACGATTCTTCCA } \\
\text { AGGCATACTACGCCTTCAAGGTCTCGTTCAACCTCTGCAT } \\
\text { CTGGGGCCTGTCGACATTCGTTGTTGCCAAGTGGGCCAG } \\
\text { ACCTCGACCCTCGCCAATGTAGTTTCGGCTGCGCTTTTG } \\
\text { GTCTCTTCTGGCAGCAGTGCGGATGGTTGGCGCACGACTT } \\
\text { TTTGCATCACCAGGTCTTCCAGGATCGTTTCTGGGGCGAT } \\
\text { CTTTTCGGTGCCTTCTTGGGAGGTGTCTGCCAGGGTTTCTC } \\
\text { ATCCTCCTGGTGGAAGGACAAGCACAACACTCACCACGCC } \\
\text { GCTCCCAACGTCCACGGTGAGGATCCCGACATTGACACTC } \\
\text { ACCCTCTGTTGACCTGGAGTGAGCATGCTCTGGAGATGTT } \\
\text { CTCGGATGTCCCTGACGAGGAGCTGACCCGCATGTGGTCG } \\
\text { CGCTTCATGGTCCTCAACCAGACCTGGTTCTACTTCCCCAT } \\
\text { TCTCTCGTTTGCCCGTCTCTCCGGTGCCTCCAGTCTATTC } \\
\text { TCTTTGTATGCCTAACGGTCAGGCCCACAAGCCCTCGGG } \\
\text { TGCGCGTGTGCCCATTTCCTTGTCGAGCAGCTGTCTCTCG } \\
\text { CTATGCACTGGACCTGGTACCTCGCCACCATGTTCCTGTC } \\
\text { GTGAAGGATCCCATCAACATGTTGTGTACTTTTGTATC } \\
\text { GCAGGCTGTTGCGGCAACTTGTTGCGCTTGTGTCTCAC } \\
\text { TCAACCACAACGGTATGCCTGTGATTCCAAGGAGGAGGC } \\
\text { AGTCGATATGGATTTCTTCACCAAGCAGATCATCACGGGT } \\
\text { CGTGATGTCCACCCTGGTCTGTTGCCAACTGGTTCACAG } \\
\text { GTGGATTGAACTACCAGATTGAGCATCACTTGTTCCCTTC } \\
\text { GATGCCCCGCCACAACTTTTCAAAGATCCAGCCTGCTGTC } \\
\text { GAGACCCTGTGCAAAAAGTACAATGTCCGATACCACACC } \\
\text { ACCGGCATGATTGAGGGAACTGCAGAGGTCTTTAGCCGTC } \\
\text { TGAACGAGGTCTCCAGGGCTGCCTCCAAGATGGGCAAGG } \\
\text { CACAGTAA }\end{array}$ \\
\hline
\end{tabular}


2. I am very intrigued by the temperate result. How do the temperature shifts affect the distribution of fatty acyl chains. Ideally, the authors would provide results similar to table 2 for the po1f control.

Answer: Thank you for your suggestion. We have provided results similar to table 2 for the po1f control as table3 (marked in red).

Table 3 Fatty acid profiles of the control strain Yarrowia lipolytica Polf under various temperature control strategies.

\begin{tabular}{ccccccc}
\hline \multirow{2}{*}{ Temperature $\left({ }^{\circ} \mathrm{C}\right)$} & \multicolumn{7}{c}{ Fatty acid composition (\%) } \\
\cline { 2 - 7 } & $\mathrm{C} 16: 0$ & $\mathrm{C} 16: 1$ & $\mathrm{C} 18: 0$ & $\mathrm{C} 18: 1$ & $\mathrm{C} 18: 2$ & $\mathrm{C} 20: 0$ \\
\hline 15 & 29.12 & 6.23 & 17.65 & 24.10 & 19.23 & 3.67 \\
20 & 34.02 & 5.03 & 18.25 & 22.54 & 17.19 & 3.05 \\
28 & 40.62 & 4.12 & 23.27 & 18.39 & 11.23 & 2.56 \\
28 (0-24h) 20 (after 24h) & 31.21 & 6.02 & 19.99 & 24.51 & 16.46 & 1.81 \\
28 (0-48h) 20 (after 48h) & 34.25 & 4.83 & 22.46 & 21.95 & 13.62 & 2.89 \\
$28(0-24 \mathrm{~h}) 15($ after 24h) & 33.12 & 4.67 & 21.45 & 23.89 & 14.19 & 2.68 \\
$28(0-48 \mathrm{~h}) 15$ (after 48h) & 35.31 & 3.89 & 20.96 & 23.25 & 13.17 & 3.42 \\
\hline
\end{tabular}

3. The authors should avoid auxotropic strains. Have they looked at the prototrophic parent? Are the results the same?

Answer: Thank you for your suggestion. Auxotropic strains have not looked at the prototrophic parent. Recently, leucine auxotrophy has been reported to affect lipogenesis of Yarrowia lipolytica because leucine may be an intracellular trigger to stimulate lipogenesis. The control and engineered strain in this study are all leucine auxotrophic strains. We think the two strains devoid of leucine can eliminate the effects of Leucine defect. Although the leucine auxotrophic strains were used in this research, compared to the original Polf, the engineered strain performed a higher glucose uptake and biomass production.

On the other hand, we are working on GLA synthesis of the engineered strain by addition of leucine stimulation. Maybe in the future we can reveal the link between leucine and GLA synthesis.

4. As the review form asks the question, I do not think the highlights accurately reflect the contributions of the manuscript. In particular, expression of the desaturase was previously done. The contribution of the present paper was to show the beneficial effect of temperature shifts.

Answer: Thank you for your suggestion. We have rewritten the highlights to reflect the beneficial effect of temperature shifts (marked in red).

\section{Highlights}

- A recombinant Y. lipolytica for GLA production was constructed. 
- The effect of temperature on production of GLA by the recombinant strain was investigated.

- DCW, lipid and GLA production were enhanced by temperature shift strategies.

- The best culture condition was $28{ }^{\circ} \mathrm{C}$ followed by 6 -day culture at $20{ }^{\circ} \mathrm{C}$. 


\section{Highlights}

- A recombinant $Y$. lipolytica for GLA production was constructed.

- The effect of temperature on production of GLA by the recombinant strain was investigated.

- DCW, lipid and GLA production were enhanced by temperature shift strategies.

- The best culture condition was $28^{\circ} \mathrm{C}$ followed by 6 -day culture at $20^{\circ} \mathrm{C}$. 


\section{Engineering Yarrowia lipolytica for efficient $\gamma$-linolenic acid production}

Mei-Li Sun ${ }^{1}$, Catherine Madzak ${ }^{2}$, Hu-Hu Liu ${ }^{1}$, Ping Song ${ }^{1,5}$, Lu-Jing Ren ${ }^{1,5}$, He Huang $^{3,4,5}$, Xiao-Jun Ji ${ }^{1,5 *}$

1. College of Biotechnology and Pharmaceutical Engineering, Nanjing Tech

University, No. 30 South Puzhu Road, Nanjing 211816, Peo`ple’s Republic of China

2.GMPA, AgroParisTech, INRA, Université Paris-Saclay, 78850, Thiverval-Grignon,

France

3. School of Pharmaceutical Sciences, Nanjing Tech University, No. 30 South Puzhu Road, Nanjing 211816, People's Republic of China

4. State Key Laboratory of Materials-Oriented Chemical Engineering, Nanjing Tech University, No. 5 Xinmofan Road, Nanjing 210009, People’s Republic of China

5. Jiangsu National Synergetic Innovation Center for Advanced Materials (SICAM), No. 5 Xinmofan Road, Nanjing 210009, People’s Republic of China

*Corresponding authors: Tel: +86 25 58139942; Fax: +86 2558139389

E-mail: xiaojunji@njtech.edu.cn (X.-J. Ji) 


\section{Abstract:}

$\gamma$-linolenic acid (GLA) has various well-documented beneficial physiological effects and high biological significance. Because the natural supply of GLA is insufficient, microbial GLA production is a promising method for pharmaceutical and nutraceutical purposes. To establish and develop a biotechnological process for GLA production by Yarrowia lipolytica, the codon-optimized $\Delta 6$-desaturase from Mortierella alpina was introduced into this yeast under the control of the strong hp4d promoter. A recombinant $Y$. lipolytica strain was constructed, which produced $4.6 \%$ GLA in total fatty acids. By using a temperature-shift strategy of cultivation, consisting in preliminary growth at $28{ }^{\circ} \mathrm{C}$ followed by 6-day culture at $20{ }^{\circ} \mathrm{C}$, optimal levels of dry cell weight (DCW), lipid content and GLA concentration were obtained from the recombinant strain: $18.55 \mathrm{~g} / \mathrm{L}, 1.16 \mathrm{~g} / \mathrm{L}$ and $71.6 \mathrm{mg} / \mathrm{L}$, respectively. These DCW, lipid and GLA values were respectively $25.7 \%, 19.6 \%$ and $60.9 \%$ higher than those obtained in the control cultivation experiment at the standard constant temperature of $28{ }^{\circ} \mathrm{C}$. This work demonstrates the excellent capacity of $Y$. lipolytica for GLA production, by combining metabolic engineering with a temperature-shift strategy.

\section{Keywords:}

$\gamma$-linolenic acid, Yarrowia lipolytica, $\Delta 6$-desaturase, Metabolic engineering, Temperature-shift strategy 


\section{Introduction}

Polyunsaturated fatty acids (PUFAs) are important metabolites which have great potential in pharmaceutical and nutraceutical applications. They are significant cellular components conferring fluidity to membranes and influencing signaling process in living cells [1,2]. As a consequence, the demand for PUFAs, for use as health supplements, has continually increased in recent decades [3]. Although PUFAs can be isolated from natural resources, such as plant seeds and marine organisms, the productivity of many of these systems is extremely low due to long growth cycles, to dependence on climate and weather variations (e.g. drought), and to expanding marine pollution. It is therefore highly desirable to produce PUFAs from alternative sources that are economical and sustainable. Nevertheless, economic production of targeted PUFA utilizing microorganisms will require optimizing native regulatory networks or assembling heterologous metabolic pathways, which can only be achieved through molecular, genetic and combinatorial approaches [4].

In recent decades, the non-conventional yeast Yarrowia lipolytica has been an attractive system for microbial oil production and has been used in a broad range of industrial applications, such as organic acid production, protein production and bioremediation [5-7]. The use of Y. lipolytica also benefits from a fully sequenced genome [8] and well-developed genetic tools [9-11], therefore, engineering this yeast can be achieved with relative ease. Moreover, $Y$. lipolytica has also been shown to be rather robust and able to grow on a variety of substrates [7].

The metabolic pathways for de novo fatty acids synthesis from glucose in $Y$. lipolytica are beginning to be thoroughly understood, as illustrated in Fig. 1. PUFA 
biosynthesis is generally associated with a variety of pathways, catalyzed by a complex series of desaturation and elongation steps. All the genes encoding desaturation and elongation activities involved in PUFA biosynthesis have been cloned and identified from multiple organisms, and the availability of these enzymes made it possible to accumulate tailored fatty acids in heterologous hosts [12]. The $\Delta 6$-fatty acid desaturase is the rate-limiting enzyme in the biosynthesis of PUFAs, and is responsible for the conversion of the essential fatty acids linoleic acid (LA, $\Delta^{9}$, $\left.{ }^{12} \mathrm{C} 18: 2\right)$ and $\alpha$-linolenic acid (ALA, $\left.\Delta^{9,12,15} \mathrm{C} 18: 3\right)$ to $\gamma$-linolenic acid (GLA, $\Delta^{6,9,12}$ C18:3) and stearidonic acid (STA, $\left.\Delta^{6,9,12,15} \mathrm{C} 18: 3\right)$, respectively. Many physiological and pathological diseases related to low levels of PUFA can be efficiently alleviated by dietary supplementation of GLA [1]. Moreover, it has been reported that GLA is unique among the (n-6) PUFA family members (LA, GLA, arachidonic acid) in its potential to suppress tumor growth and metastasis [2]. These beneficial effects made GLA a much valuable and sought-after product. The principal sources for GLA are vegetable oils derived from evening primrose, borage and blackcurrant. However, given the finite supply of arable land and increasing demand for GLA, it is unlikely that GLA from plant oils will contribute significantly to meeting future food supplementation needs.

The most significant unsaturated fatty acids produced by $Y$. lipolytica are oleic acid (C18:1) and linoleic acid (C18:2), whereas unsaturated fatty acids with more than two double bonds, so-called PUFAs, are not synthesized by this microorganism naturally [13]. However, Y. lipolytica has been proposed as a preferred host for PUFA 
production [14], and engineering of PUFA biosynthesis pathways in this yeast has already been attempted. Xue et al [15] produced tailored fatty acids comprising eicosapentaenoic acid (EPA, C22:5) at 15\% of dry cell weight (DCW) by metabolic engineering of $Y$. lipolytica and commercial products have been developed using this recombinant yeast [16]. Zhang et al. [17] successfully produced trans-10, cis-12 conjugated linoleic acid in $Y$. lipolytica Po1h strain via expression of linoleic acid isomerase. Efforts towards effective carbon flow redistribution of lipid synthesis pathways in this promising yeast should continue to meet the increasing demand.

A previous work has reported construction of a de novo GLA biosynthesis system in Y. lipolytica via expression of desaturases cloned from Mortierella alpina [18]. In the present study, we planned to improve GLA production in $Y$. lipolytica by expression of a codon-optimized $\Delta 6$-desaturase from M. alpina under the control of the $\mathrm{hp} 4 \mathrm{~d}$ promoter and to optimize fermentation conditions. Temperature is one of the principal factors that affect cell growth and fatty acid synthesis. However, little attention has been paid up to now to define the effects of temperature on GLA fermentation by $Y$. lipolytica and to study a two-stage process that uses different temperatures to optimize cell growth and GLA fermentation. In an attempt to enhance GLA production, fermentation conditions were modified using a temperature-shift strategy to redistribute carbon flux from saturated to unsaturated fatty acids in $Y$. lipolytica. By combining metabolic engineering with a temperature-shift strategy, GLA production was achieved in Y. lipolytica at levels of up to $6.1 \%$ of total fatty acids and $18.55 \mathrm{~g} / \mathrm{L}$ DCW. To our best knowledge, this work represents the first effort 
to utilize a temperature-shift strategy for the production of GLA in this yeast and may provide alternative routes to lower production costs in future industrial applications.

\section{Materials and Methods}

\subsection{Strains, plasmids and culture conditions}

E. coli DH5 $\alpha$ was grown in Luria-Bertani broth containing ampicillin (100 $\mu \mathrm{g} / \mathrm{mL}$ ). The auxotrophic strain Y. lipolytica Po1f (ATCC \# MYA-2613, MatA, leu2-270, ura3-302, xpr2-322, axpl-2/Ura-, Leu') and the hp4d-based expression vector p0, carrying $U R A 3$ selection marker, have been described previously [19, 20].

Peptone dextrose culture medium (YPD) containing $20 \mathrm{~g} / \mathrm{L}$ peptone, $10 \mathrm{~g} / \mathrm{L}$ yeast extract and $20 \mathrm{~g} / \mathrm{L}$ glucose was used for normal growth. The synthetic minimal medium, minus uracil (YSU-Ura), plates for the selection of transformed Y. lipolytica were made with $6.7 \mathrm{~g} / \mathrm{L}$ yeast nitrogen base (without amino acids), $0.77 \mathrm{~g} / \mathrm{L}$ CSM-Ura (Complete Supplement Mixture minus uracil), $20 \mathrm{~g} / \mathrm{L}$ glucose and $20 \mathrm{~g} / \mathrm{L}$ agar. The shake flask fermentation experiments were carried out in a medium comprising $8 \mathrm{~g} / \mathrm{L}$ yeast nitrogen base (without amino acids), $9 \mathrm{~g} / \mathrm{L}$ yeast extract, 80 $\mathrm{g} / \mathrm{L}$ glucose and $10 \mu \mathrm{g} / \mathrm{L}$ biotin. From an agar plate, a single colony of yeast was inoculated into YPD medium (100 mL in $500 \mathrm{~mL}$ flask, $\left.200 \mathrm{rpm}, 28{ }^{\circ} \mathrm{C}, 48 \mathrm{~h}\right)$ and grown to an optical density $\left(\mathrm{OD}_{600}\right)$ of 4.0-5.0. The pre-cultures were allowed to grow in fermentation medium with an inoculum size of $10 \%(\mathrm{v} / \mathrm{v})$, for a total duration of 7 days, during which aliquots were taken and biomass, lipid content and fatty acid compositions analyzed.

\subsection{Plasmid construction and transformation}

Standard molecular genetic techniques were used throughout this study [21]. 
Recombinant vectors that express the gene of interest under the control of the hp4d promoter were generated using the ClonExpress ${ }^{\mathrm{TM}}$ MultiS one step cloning kit (Vazyme, China), according to the manufacturer's instructions.

The expression vector for the $\Delta 6$-fatty acid desaturase gene was constructed as follows. The $\Delta 6$-fatty acid desaturase gene from Mortierella alpine was codon optimized for expression in $Y$. lipolytica. The sequences for $\Delta 6$-fatty acid desaturase and codon-optimized $\Delta 6$-fatty acid desaturase were listed in Supplementary Table 1. The codon-optimized $\Delta 6$-fatty acid desaturase gene was then amplified using the following primers:

\section{D6-F (5'-CAGTGGCCGGATCCGAATTCATGGCCGCCGCCCCCTCCGT-3’)}

D6-R (5'-GCGGCCGCCATATGGAATTCTTACTGAGCCTTACCCATCT-3')

with the underlined nucleotides representing added EcoRI restriction sites.

The PCR-amplified sequences were digested using EcoRI and one-step cloned into the EcoRI-digested p0 backbone to generate the recombinant vector p0-6-D. The newly constructed plasmid was screened by EcoRI digestion and PCR with the primers D6-F and D6-R, and confirmed by DNA sequencing.

Subsequently, the plasmid was linearized by digestion and integrated into Po1f genome, at the $X P R 2$ terminator locus, using the lithium acetate transformation method [22]. Transformants of the resulting strain Po1f-6-D were selected on YSC-Ura plates. To verify the transformants for correct integration, genomic DNA was extracted and used as template for PCR analysis. Verified transformants were stored for long term at $-80{ }^{\circ} \mathrm{C}$ in glycerol solution and for short-term at $4{ }^{\circ} \mathrm{C}$ on 
YSC-Ura plates.

\subsection{Analytical methods}

The concentration of extracellular glucose was measured by a biosensor equipped with a glucose oxidase electrode (SBA-40C, Institute of Biology, Shandong Academy of Sciences, China). $\mathrm{OD}_{600}$ were acquired by monitoring the optical density of the cultures at $600 \mathrm{~nm}$ using a UV-visible spectrophotometer (Lambda-25, Perkin-Elmer, USA) with appropriate sample dilution. DCW determination was performed by harvesting the culture broth, after which the cells were separated by centrifugation at $5000 \mathrm{rpm}$ for 6 min and washed with distilled water. Subsequently, the supernatant was discarded and the cell mass was dried at $65{ }^{\circ} \mathrm{C}$ to constant weight.

\subsection{Lipid extraction and fatty acid analysis}

Lipids from an appropriate weighted aliquot of dried cells were extracted using the procedure by Folch et al [23]. A measured quantity of dried cells (roughly $0.5 \mathrm{~g}$ ) was homogenized in $5 \mathrm{~mL}$ of $4 \mathrm{M} \mathrm{HCl}$ at room temperature for $30 \mathrm{~min}$, resuspended in a mixture of $10 \mathrm{~mL}$ chloroform and $5 \mathrm{~mL}$ methanol and vortexed for $30 \mathrm{~min}$. The lipids were separated by evaporating the chloroform.

The extracted total lipids were methylated to generate fatty acid methyl esters (FAMEs) as follows: quantitative lipid extracts (roughly $5 \mathrm{mg}$ ) were methylated by adding $1 \mathrm{~mL}$ hexane and $100 \mu \mathrm{L}$ methanol, after which the samples were vortexed for $10 \mathrm{~min}$ and centrifuged for $3 \mathrm{~min}$ at $3000 \mathrm{~g}$. An aliquot comprising $800 \mu \mathrm{L}$ of the hexane layer was then transferred into a clean $1.5 \mathrm{~mL}$ microcentrifuge tube for Gas 
Chromatography-Flame Ionization Detector (GC-FID) analysis. Samples of $1 \mu \mathrm{L}$ were injected into a GC-FID (Agilent 6890 GC), equipped with an Agilent HP-5 column, with detector temperature set to $250{ }^{\circ} \mathrm{C}$ and $\mathrm{N}_{2}$ flow at $1 \mathrm{~mL} / \mathrm{min}$. Fatty acid compositions were determined by comparing peak retention times to those of known standard substances. The amount of fatty acids was quantified by comparing the peak area of the analyte to the peak of the standard substance at known concentrations.

\section{Results}

\subsection{Construction of the recombinant GLA producing strain}

The DNA sequence of the $\Delta 6$-fatty acid desaturase from $M$. alpina was redesigned using codons favored by $Y$. lipolytica, and the GC content was adjusted to that of the host. The codon adaption index (CAI) was improved from 0.83 (natural sequence) to 0.91 (synthetic codon gene). The single-copy expression vector $\mathrm{p} 0$, which places the gene of interest under the control of the strong quasi-constitutive hybrid hp4d promoter [19], was used to develop the recombinant strain. To produce GLA in $Y$. lipolytica, the codon-optimized $\Delta 6$-desaturase gene from $M$. alpina was used to construct the recombinant vector p0-6-D bearing the desaturase expression cassette. The entire process of constructing the recombinant strain Po1f-6-D and verification of GLA production in the recombinant strain is shown in Fig. 2.

Based on the plasmid p0 (URA3), the recombinant plasmid p0-6-D was constructed as shown in Fig. 2A. The recombinant plasmid was digested with AvrII and used to transform Y. lipolytica Polf strain (Fig. 2B). The integration of a single copy of p0-6-D at the XPR2 terminator genomic site of Po1f allowed cell growth of 
the corresponding transformants on Uracil-deficient plates after 2-3 days of incubation. Subsequently, correct plasmid integration was verified by PCR, as described in the Materials and Methods section (Fig. 2C). To determine whether codon-optimized $\Delta 6$-desaturase could be expressed effectively in $Y$. lipolytica, the engineered strain Po1f-6-D was incubated in fermentation medium (Fig. 2D). When lipids were extracted and fatty acid profiles were analyzed by GC, it was observed that GLA represented $4.6 \%$ of total fatty acids in the engineered strain Polf-6-D, when it was not detected in the control strain Po1f (Fig. 2E). These results indicate an efficient conversion of the endogenous linoleic acid to GLA, catalyzed by the heterologous $\Delta 6$-desaturase.

The fermentation performance of the engineered GLA-producing strain Po1f-6-D was comparable to (and even slightly better than) that of the control strain Po1f, as shown in Fig. 3. The value of DCW increased in the engineered strain compared to the control, reaching a maximum of $14.84 \mathrm{~g} / \mathrm{L}$ (Fig. 3A). Consistently with the increased cell growth, the glucose uptake rate was also higher in the engineered strain (Fig. 3B). The lipid yield, which attained $6.55 \%$ in the engineered strain, was not significantly different from that of the control strain at $6.23 \%$. The final fatty acid profiles of the two strains are shown in Fig. 3C. Concomitantly with the appearance of GLA in the engineered strain, there was a slight decrease in linoleic acid and oleic acid production. No other significant difference in the final lipid profiles of the two strains was documented, with palmitic acid, stearic acid and oleic acid being the most abundant fatty acid species and accounting for roughly 38\%, 23\% and 18\%, 
respectively.

\subsection{Optimization of GLA synthesis by using a temperature-shift strategy}

The engineered strain $Y$. lipolytica Polf-6-D, obtained by expressing a codon-optimized $\Delta 6$-desaturase, produced a certain amount of GLA ( $4.6 \%$ of total fatty acid) under standard growth conditions at $28{ }^{\circ} \mathrm{C}$. Starting from this result, efforts were continued to improve the production of GLA by Po1f-6-D, which can be achieved by enhancing either DCW or intracellular GLA proportion. It is known that GLA formation entails a complex combination of different reactions, and both cell growth and glucose utilization are important for GLA production. It has been reported that the maximal growth temperature of $Y$. lipolytica does not exceed $32-34{ }^{\circ} \mathrm{C}$ [24]. In the present study, cell growth was severely inhibited above $35^{\circ} \mathrm{C}$ (data not shown). Y. lipolytica Po1f-6-D was consequently cultivated at $15{ }^{\circ} \mathrm{C}, 20{ }^{\circ} \mathrm{C}$ and $28{ }^{\circ} \mathrm{C}$. As illustrated in Fig. 4A, cultivation at $28{ }^{\circ} \mathrm{C}$ led to the highest value of DCW, namely $14.84 \mathrm{~g} / \mathrm{L}$, compared to $13.34 \mathrm{~g} / \mathrm{L}$ at $20^{\circ} \mathrm{C}$ and $11.57 \mathrm{~g} / \mathrm{L}$ at $15^{\circ} \mathrm{C}$. In accordance with increasing cell growth at higher temperature, the residual glucose level decreased between $15^{\circ} \mathrm{C}$ and $28{ }^{\circ} \mathrm{C}$ (Fig. 4B). There was almost $30 \mathrm{~g} / \mathrm{L}$ glucose left in the culture broth at $15{ }^{\circ} \mathrm{C}$, while the initial glucose concentration was $80 \mathrm{~g} / \mathrm{L}$. A comparison of GLA concentrations at different temperatures is shown in Fig. 4C: it was generally higher at lower temperatures. The maximum GLA ratio was $7.99 \%$ of total fatty acids at $15{ }^{\circ} \mathrm{C}$ and a relatively high GLA ratio of $6.91 \%$ was achieved at $20{ }^{\circ} \mathrm{C}$ (Table 2), whereas the maximum value of GLA concentration $(57.82 \mathrm{mg} / \mathrm{L})$ was achieved at $20{ }^{\circ} \mathrm{C}$ (Fig. 4C) due to the obvious cell growth inhibition at $15{ }^{\circ} \mathrm{C}$. 
These results are in line with the situation in other eukaryotic microorganisms, such as $M$. alpina, in which a relatively high temperature enhanced cell growth, and a lower temperature was more favorable for PUFA synthesis [25].

Although a lower temperature is more favorable for increased GLA synthesis, it is disadvantageous for practical reasons, since it results in low biomass concentration. As shown in Fig. 5A, controlled temperature-shift processes were developed, to take into account the differences of temperatures suitable for cell growth or GLA synthesis: a temperature of $28{ }^{\circ} \mathrm{C}$ at an earlier fermentation stage was favorable to cell growth, and shifts to $20{ }^{\circ} \mathrm{C}$ or $15{ }^{\circ} \mathrm{C}$ were done after 1 or 2 days of growth at $28{ }^{\circ} \mathrm{C}$. The effects of these temperature-shift strategies on GLA concentration, glucose consumption and DCW are shown in Fig. 5B to 5E. The GLA concentration and DCW of cultures grown in the four temperature-shift experiments were all higher than in the control at $28{ }^{\circ} \mathrm{C}$. The improvement in biomass concentration was accompanied by increased glucose consumption. The highest GLA content of $6.1 \%$ and the highest DCW of $18.78 \mathrm{~g} / \mathrm{L}$ were achieved with the shift to $20{ }^{\circ} \mathrm{C}$ after 1 day. An interesting phenomenon was also observed when the shift was done at 2 days, in the early stationary phase: although a longer growth phase at optimal temperature would have been expected to be beneficial, the accumulation of GLA and biomass concentration were in fact lower than when the shift was done at day 1 , during the $\log$ phase (Fig. 5). A comparison of the fermentation data obtained using the different temperature control strategies is summarized in Table 1.The best temperature-shift strategy (shift from 28 to $20{ }^{\circ} \mathrm{C}$ after 1 day) considerably improved the $\mathrm{DCW}$, total 
lipids and GLA concentration, which were increased by $25.7 \%, 19.6 \%$ and $60.9 \%$, respectively compared to standard cultivation at $28{ }^{\circ} \mathrm{C}$. Table 2 and 3 also showed some interesting variations of fatty acid profiles of po1f-6-D and po1f in cells under different temperature control strategies. Here, the best result in term of GLA ratio, namely $6.17 \%$, obtained by using temperature shifting strategies, does not have a competitive edge over the result of $7.99 \%$ previously obtained at $15{ }^{\circ} \mathrm{C}$. However, in order to develop a viable process, we should not take into account only the GLA percentage, but also the DCW and total lipid amount.

\section{Discussion}

The aim of this study was to construct a de novo GLA biosynthesis pathway by transforming the yeast $Y$. lipolytica with a codon-optimized $\Delta 6$-desaturase from $M$. alpina. The bulk of the isolated fatty acids were palmitic acid, palmitoleic acid, stearic acid, oleic acid, linoleic acid and arachidic acid in the control strain $Y$. lipolytica Po1f. We choose to produce GLA in Y. lipolytica instead of using the model microorganism Saccharomyces cerevisiae, because $Y$. lipolytica is an oleaginous yeast with the innate ability to accumulate linoleic acid, a precursor of GLA. To date, several groups have been able to produce low amounts of GLA in S. cerevisiae via heterologous expression of $\Delta 6$-desaturases, but supplementation with linoleic acid as exogenous substrate was necessary [26, 27].

Chuang et al. [18] previously used a single-copy integrative vector with hp4d promoter to express a $\Delta 6$-desaturase, cloned from M. alpine, in Y. lipolytica. In our study, a codon-optimized $\Delta 6$-desaturase was expressed under the control of the same hp4d promoter. When the codon-optimized $\Delta 6$-desaturase integrant was cultivated on 
glucose in shake flasks at $28{ }^{\circ} \mathrm{C}$, GLA was synthesized in the engineered strain in the absence of exogenously added fatty acid substrates and represented $4.6 \%$ of total lipids, a level similar to the $4.9 \%$ found in the work of Chuang et al. [18]. Therefore, it seems that the fact of using in our study a synthetic gene, adapted to $Y$. lipolytica codon bias and GC content, the two expression systems being otherwise similar, is not particularly beneficial.

During the time course of GLA fermentation, the engineered strain Po1f-6-D completed exponential growth by hour 48, after which DCW remained relatively constant. In addition to GLA synthesis, the biomass from the engineered strain was significantly higher than in the control and glucose was consumed more rapidly, suggesting that the expression of $\Delta 6$-desaturase elevated the glucose uptake rate and somewhat favored the growth of $Y$. lipolytica. This result was different from a previous work in which overexpression of acetyl-CoA carboxylase (ACC1) and diacylglycerol acyltransferase (DGA1), allowing to improve lipid content from $8.7 \%$ to $41.4 \%$, impaired growth in Y. lipolytica [28]. Thus, expression of ACC1 and DGA1 impaired cell growth, while our Po1f-6-D engineered strain consumed glucose more rapidly than the control, because it diverted more cellular resources toward oil synthesis instead of synthesizing non-lipid biomass. The integration of $\Delta 6$-desaturase also resulted in a higher glucose uptake than in control strain. However, more cellular resources were diverted toward non-lipid biomass instead of lipogenesis when overall lipid content did not change. This might be the reason why Po1f-6-D engineered strain reached a higher biomass concentration than the control strain. 

fatty acids). In order to optimize GLA synthesis, we took advantage of the high sensitivity of fatty acid desaturases to temperature change and higher activity of the enzymes at lower growth temperatures [29]. A relatively high temperature was favorable for glucose consumption and biomass accumulation, but a lower temperature was beneficial for GLA synthesis. This may be due to distinct rate-limiting reactions which rely on temperature, resulting in a better utilization of carbon and/or energy at the suitable temperature and in increased availability of vital coenzymes/cofactors (e.g. ATP, NADPH) for either cell reproduction or production of metabolites [30]. Our results illustrate that a low culture temperature favored unsaturated fatty acid accumulation during GLA fermentation (Table 2 and 3). In order to maintain a proper membrane lipid fluidity and function, the proportions of unsaturated fatty acids, including GLA, increase in response to low cultivation temperature [31]. On the other hand, it is also known that dissolved oxygen (DO) level is lower at higher temperatures. Thus, at lower temperature a greater amount of molecular oxygen is available that allows the oxygen-dependent enzymes to catalyze fatty acid desaturation [32]. In the present study, the changes in GLA yield at different temperatures might be due to a combination of all of the factors above.

It has been reported that intracellular lipid accumulation increases following depletion of nitrogen from the medium (when DCW reaches its maximum value and remains relatively constant), and the biochemical mechanisms leading to fatty acid biosynthesis after nitrogen exhaustion have been clarified [33, 34]. Thus, lipogenesis 
continues throughout the fermentation process, despite cessation of biomass accumulation within 2 days. When metabolite production is not dependent on cell growth but is affected by culture temperature, a novel two-stage temperature cultivation strategy could be applied to maximize the level of the target metabolite. The idea was to achieve optimal cell reproduction and GLA production by the sequential use of separately optimized temperatures.

Thus, we developed a temperature-shift strategy for the production of GLA in $Y$. lipolytica and determined the optimal time-shift point by lowering the temperature (from $28^{\circ} \mathrm{C}$ to $15^{\circ} \mathrm{C}$ or $20^{\circ} \mathrm{C}$ ) during the $\log$ phase (shift after day 1 ) or in the early stationary phase (shift after day 2). Interestingly, the GLA ratio and biomass concentration were higher with a shift after day 1 , suggesting that cells have to be shifted to lower temperature at a certain point in the log phase to produce a high level of GLA. This result is in good agreement with previous studies which have shown that cells shifted to a lower temperature during the log phase have the potential to produce a higher level of ricinoleic acid [35]. Zheng et al. [36] demonstrated specific cell growth rate are higher in the log phase than that of in the stationary phase. Thus, the cells shifted to lower temperature in the log phase were competent to produce a high level of GLA as described in the work of R. Holic et al. [35]. The highest total lipid and GLA concentrations of $1.16 \mathrm{~g} / \mathrm{L}$ and $71.6 \mathrm{mg} / \mathrm{L}$ were obtained from cells shifted to $20{ }^{\circ} \mathrm{C}$ after day 1 . However, the GLA portions of total fatty acids obtained with the temperature-shifts remain lower than those from corresponding single temperature cultures at $20^{\circ} \mathrm{C}$ or $15{ }^{\circ} \mathrm{C}$ (Table 2), which infers that increased biomass 
is probably a main contributor to increased GLA concentration in the temperature-shift experiments.

In the present study, the optimal biomass concentration, total lipids and GLA concentration were $18.55 \mathrm{~g} / \mathrm{L}, 1.16 \mathrm{~g} / \mathrm{L}$ and $71.6 \mathrm{mg} / \mathrm{L}$ under the conditions of $28{ }^{\circ} \mathrm{C}$ for 1 day followed by $20{ }^{\circ} \mathrm{C}$ for 6 days, which represents increases of $25.7 \%, 19.6 \%$ and $60.9 \%$, respectively, compared to standard cultivation at $28{ }^{\circ} \mathrm{C}$. Moreover, this optimal GLA yield still represents an increase of $24.7 \%$ when compared to the best result obtained in an experiment at a single temperature, namely $20^{\circ} \mathrm{C}$, at which GLA production was favored despite low cell growth. It appears that cell growth and GLA accumulation could be optimized simultaneously by applying this temperature-shift strategy, as opposed to the single temperature controls in which lipid accumulation was in conflict with biomass concentration. Higher dissolved oxygen levels at lower temperatures were maybe also able to mitigate cell autolysis at later stages of fermentation [30]. This might be the reason why the biomass concentration did not decrease but actually increased when the culture temperature was shifted to a lower value after 24 or $48 \mathrm{~h}$ of growth.

\section{Conclusions}

In conclusion, a de novo GLA biosynthesis system was successfully constructed by transforming $Y$. lipolytica with a codon-optimized $\Delta 6$-desaturase gene from $M$. alpina. The GLA concentration in the engineered strain was further increased by applying a temperature-shift cultivation strategy and reached $6.1 \%$ from total fatty acids. Similarly, the two-stage culture strategy proposed here, based on Y. lipolytica fermentation characteristics, could be applied to improving production of many other 
metabolites.

\section{Acknowledgements}

This work was financially supported by the National Science Foundation for Distinguished Young Scholars of China (No. 21225626), the National Natural Science Foundation of China (Nos. 21376002 and 21476111), Jiangsu Provincie Natural Science Foundation of China (No. BK20131405), the National High-Tech R\&D Program of China (No. 2014AA021703), and the Priority Academic Program Development of Jiangsu Higher Education Institutions. 


\section{References}

[1] D.F. Horrobin, Nutritional and medical importance of gamma-linolenic acid, Prog. Lipid Res. 31 (1992) 163-194.

[2] Y.Y. Fan, R.S. Chapkin, Importance of dietary gamma-linolenic acid in human health and nutrition, J. Nutr. 128 (1998) 1411-1414.

[3] D. Xie, E.N. Jackson, Q. Zhu, Sustainable source of omega-3 eicosapentaenoic acid from metabolically engineered Yarrowia lipolytica: from fundamental research to commercial production, Appl. Microbiol. Biotechnol. 99 (2015) 1599-1610.

[4] Q. Zhu, E.N. Jackson, Metabolic engineering of Yarrowia lipolytica for industrial applications, Curr. Opin. Biotechnol. 36(2015) 65-72.

[5] G. Barth, C. Gaillardin, Physiology and genetics of the dimorphic fungus Yarrowia lipolytica, FEMS Microbiol. Rev. 19 (1997) 219-237.

[6] S. Papanikolaou, L. Muniglia, I. Chevalot, G. Aggelis, I. Marc, Yarrowia lipolytica as a potential producer of citric acid from raw glycerol, J. Appl. Microbiol. 92 (2002) 737-744.

[7] H.H. Liu, X.J. Ji, H. Huang, Biotechnological applications of Yarrowia lipolytica: past, present and future, Biotechnol. Adv. 33 (2015) 1522-1546.

[8] L. Liu, H.S. Alper, Draft genome sequence of the oleaginous yeast Yarrowia lipolytica Po1f, a commonly used metabolic engineering host, Genome Announc. 2 (2014) e00652-14.

[9] C. Madzak, Yarrowia lipolytica: recent achievements in heterologous protein expression and pathway engineering, Appl. Microbiol. Biotechnol. 99 (2015) 4559-4577.

[10] C.M. Schwartz, M.S. Hussain, M. Blenner, I. Wheeldon, Synthetic RNA polymerase III promoters facilitate high-efficiency CRISPR-Cas9-mediated genome editing in Yarrowia lipolytica, ACS. Synth. Biol. 5 (2016) 356-359.

[11] S. Gao, Y. Tong, Z. Wen, L. Zhu, M. Ge, D. Chen, Y. Jiang, S Yang, Multiplex gene editing of the Yarrowia lipolytica genome using the CRISPR-Cas9 system, J. Ind. Microbiol. Biot 43 (2016) 1085-1093.

[12] Y. Gong, X. Wan, M. Jiang, C. Hu, H. Hu, F. Huang, Metabolic engineering of microorganisms to produce omega-3 very long-chain polyunsaturated fatty acids, Prog. Lipid Res. 56 (2014) 19-35.

[13] K.R. Pomraning, S. Wei, S.A. Karagiosis, Y.M. Kim, A.C. Dohnalkova, B.W. Arey, E.L. Bredeweg, G. Orr, T.O. Metz, S.E. Baker, Comprehensive metabolomic, lipidomic and microscopic profiling of Yarrowia lipolytica during lipid accumulation Identifies targets for increased lipogenesis, PLoS One 10 (2015) e0123188.

[14] R. Ledesma-Amaro, J.M. Nicaud, Yarrowia lipolytica as a biotechnological chassis to produce usual and unusual fatty acids, Prog. Lipid Res. 61 (2015) 40-50.

[15] Z. Xue, P.L. Sharpe, S.P. Hong, N.S. Yadav, D. Xie, D.R. Short, H.G. Damude, 

Macool, D.H. Hollerbach, H. Zhang, D.M. Arcilla, S.A. Bledsoe, K. Croker, E.F. McCord, B.D. Tyreus, E.N. Jackson, Q. Zhu, Production of omega-3 eicosapentaenoic acid by metabolic engineering of Yarrowia lipolytica, Nat. Biotechnol. 31 (2013) 734-740.

[16] M. Groenewald, T. Boekhout, C. Neuveglise, C. Gaillardin, P.W.M. Van Dijck, M. Wyss, Yarrowia lipolytica: Safety assessment of an oleaginous yeast with a great industrial potential, Crit. Rev. Microbiol. 40 (2014) 187-206.

[17] B. Zhang, C. Rong, H. Chen, Y. Song, H. Zhang, W. Chen, De novo synthesis of trans-10, cis-12 conjugated linoleic acid in oleaginous yeast Yarrowia lipolytica, Microb. Cell. Fact. 11 (2012) 51.

[18] L.T. Chuang, D.C. Chen, J.M. Nicaud, C. Madzak, Y.H. Chen, Y.S. Huang, Co-expression of heterologous desaturase genes in Yarrowia lipolytica, N. Biotechnol. 27 (2010) 277-282.

[19] C. Madzak, B. Tréton, S. Blanchin-Roland, Strong hybrid promoters and integrative expression/secretion vectors for quasi-constitutive expression of heterologous proteins in the yeast Yarrowia lipolytica, J. Mol. Microbiol. Biotechnol. 2 (2000) 207-216.

[20] D. Swennen, M.F. Paul, L. Vernis, J.M. Beckerich, A. Fournier, C. Gaillardin, Secretion of active anti-Ras single-chain Fv antibody by the yeasts Yarrowia lipolytica and Kluyveromyces lactis, Microbiol. 148 (2002) 41-50.

[21] J. Sambrook, E.F. Fritsch, T. Maniatis, Molecular cloning, Cold spring harbor laboratory press, New York, 1989.

[22] J.W. Xuan, P. Fournier, C. Gaillardin, Cloning of the lys5-gene encoding saccharopine dehydrogenase from the yeast Yarrowia lipolytica by target integration, Curr. Genet. 14 (1988) 15-21.

[23] J. Folch, M. Lees, G.H. Sloane Stanley, A simple method for the isolation and purification of total lipides from animal tissues, J. Biol. Chem. 226 (1957) 497-509.

[24] A. Beopoulos, T. Desfougeres, J. Sabirova, S. Zinjarde, C. Neuveglise, J.M. Nicaud, The hydrocarbon-degrading oleaginous yeast Yarrowia lipolytica, in: K.N. Timmis, T.J. McGenity, J.R. Van Der Meer, V. De Lorenzo (Eds.), Handbook of Hydrocarbon and Lipid Microbiology, Springer-Verlag Berlin Heidelberg, 2010, pp. 2111-2121.

[25] C. Peng, H. Huang, X.J. Ji, X. Liu, J.Y. You, J.M. Lu, L.L. Cong, X. Xu, P.K. Ouyang, A temperature-shift strategy for efficient arachidonic acid fermentation by Mortierella alpina in batch culture, Biochen. Eng. J. 53 (2010) 92-96.

[26] H. Lu, J.N. Li, Y.R. Chai, X.K. Zhang, Identification and characterization of a novel delta 6-fatty acid desaturase gene from Rhizopus nigricans, Mol. Biol. Rep. 36 (2009) 2291-2297

[27] P. Kurdrid, S. Subudhi, A. Hongsthong, M. Ruengjitchatchawalya, M. Tanticharoen, Functional expression of Spirulina- $\Delta 6$ desaturase gene in yeast, Saccharomyces cerevisiae, Mol. Biol. Rep. 32 (2005) 215-226.

[28] M. Tai, G. Stephanopoulos, Engineering the push and pull of lipid biosynthesis 
in oleaginous yeast Yarrowia lipolytica for biofuel production, Metab. Eng. 15 (2013) 1-9.

[29] M.H.W. Weber, W. Klein, L. Muller, U.M. Niess, M.A. Marahiel, Role of the Bacillus subtilis fatty acid desaturase in membrane adaptation during cold shock, Mol. Microbiol. 39 (2001) 1321-1329.

[30] I.M. Aasen, T. Moretro, T. Katla, L. Axelsson, I. Storro, Influence of complex nutrients, temperature and $\mathrm{pH}$ on bacteriocin production by Lactobacillus sakei CCUG 42687, Appl. Microbiol. Biotechnol. 53 (2000) 159-166.

[31]A.M. Gounot, Bacterial life at low-temperature-physiological aspects and biotechnological implications, J. Bacteriol. 71 (1991) 386-397.

[32] C. Feng, M.R. Johns, Effect of $\mathrm{C} / \mathrm{N}$ ratio and aeration on the fatty acid composition of heterotrophic Chlorella-sorokiniana, J. Appl. Phycol. 3 (1991) 203-209.

[33] C. Ratledge, J.P. Wynn, The biochemistry and molecular biology of lipid accumulation in oleaginous microorganisms, Adv. Appl. Microbiol. 51 (2002) $1-51$.

[34] C. Ratledge, Fatty acid biosynthesis in microorganisms being used for Single Cell Oil production, Biochimie. 86 (2004) 807-815.

[35] R. Holic, H. Yazawa, H. Kumagai, H. Uemura, Engineered high content of ricinoleic acid in fission yeast Schizosaccharomyces pombe, Appl. Microbiol. Biotechnol. 95 (2012) 179-187.

[36] M. Zheng, G.C Du, W.F. Guo, J. Chen, A temperature-shift strategy in batch microbial transglutaminase fermentation, Process Biochem. 36 (2001) 525-530. 
Fig. 1. Synthetic pathways of fatty acids in Yarrowia lipolytica. The native fatty acid pathway is indicated in solid lines and the engineered pathway is indicated in dotted lines. TCA cycle, tricarboxylic acid cycle; FAS, fatty acid synthase; mCT, mitochondrial membrane citrate transporter; cCT, cytomembrane citrate transporter; PA, palmitic acid; SA, stearic acid; OA, oleic acid; LA, linoleic acid; GLA, $\gamma$-linolenic acid; ALA, $\alpha$-linolenic acid; STA, stearidonic acid; EDA, eicosadienoic acid; ETrA, eicosatrienoic acid; ETA, eicosatetraenoic acid; DGLA, dihomo- $\gamma$-linolenic acid; ARA, arachidonic acid; EPA, eicosapentaenoic acid; DPA, docosapentenoic acid; DHA, docosahexaenoic acid.

Fig. 2. Overview of the construction of recombinant Yarrowia lipolytica strain Po1f-6-D. (A) The recombinant plasmid p0-6-D was constructed by one step cloning. (B) p0-6-D was used to transform Po1f and the transformants were selected on YSC-Ura plates. (C) The recombinant strain was confirmed by PCR; Lane 1: the PCR fragment amplified from the control strain Polf genomic DNA; Lane M: Marker DL 10000; Lane 2: the PCR fragment amplified from the genomic DNA of engineered strain Po1f-6-D. (D) Flask fermentation of the confirmed transformant cultured in triplicate. (E) Gas chromatographic analysis of fatty acid composition in the engineered strain Po1f-6-D and the control strain Po1f. C16:0, palmitic acid; C16:1, palmitoleic acid; C18:0, stearic acid; C18:1, oleic acid; C18:2, linoleic acid; C18:3, $\gamma$-linolenic acid; C20:0, arachidic acid.

Fig. 3. Fermentation performance of the control strain Yarrowia lipolytica Po1f and the engineered strain Yarrowia lipolytica Po1f-6-D in shake flask cultures. Each strain 
was cultured in triplicate. (A) Time course of dry cell weight (DCW). (B) Time course of glucose consumption. (C) Final fatty acid distribution profiles at the time cells were harvested for DCW determination. C16:0, palmitic acid; C16:1, palmitoleic acid; C18:0, stearic acid; C18:1, oleic acid; C18:2, linoleic acid; C18:3, $\gamma$ linolenic acid; C20:0, arachidic acid.

Fig. 4. Effect of culture temperature on dry cell weight (DCW) (A), glucose (B) and $\gamma$-linolenic acid (GLA) concentration (C) during GLA fermentation by the engineered Yarrowia lipolytica strain Po1f-6-D.

Fig. 5. Effect of a temperature shift from $28{ }^{\circ} \mathrm{C}$ to $20^{\circ} \mathrm{C}$ or $15^{\circ} \mathrm{C}$ of the engineered Yarrowia lipolytica strain Po1f-6-D at log and stationary phases. (A) Schematic diagram of temperature-shift experiments performed with the engineered strain Po1f-6-D. (B to E) Time course of glucose consumption, dry cell weight (DCW) and $\gamma$-linolenic acid (GLA) concentration of the engineered strain Po1f-6-D with temperature shift from $28{ }^{\circ} \mathrm{C}$ to $15{ }^{\circ} \mathrm{C}$ after day 1 (B), $28{ }^{\circ} \mathrm{C}$ to $20{ }^{\circ} \mathrm{C}$ after day 1 (C), $28^{\circ} \mathrm{C}$ to $15^{\circ} \mathrm{C}$ after day 2 (D) and shift from $28^{\circ} \mathrm{C}$ to $20^{\circ} \mathrm{C}$ after day 2 (E). 


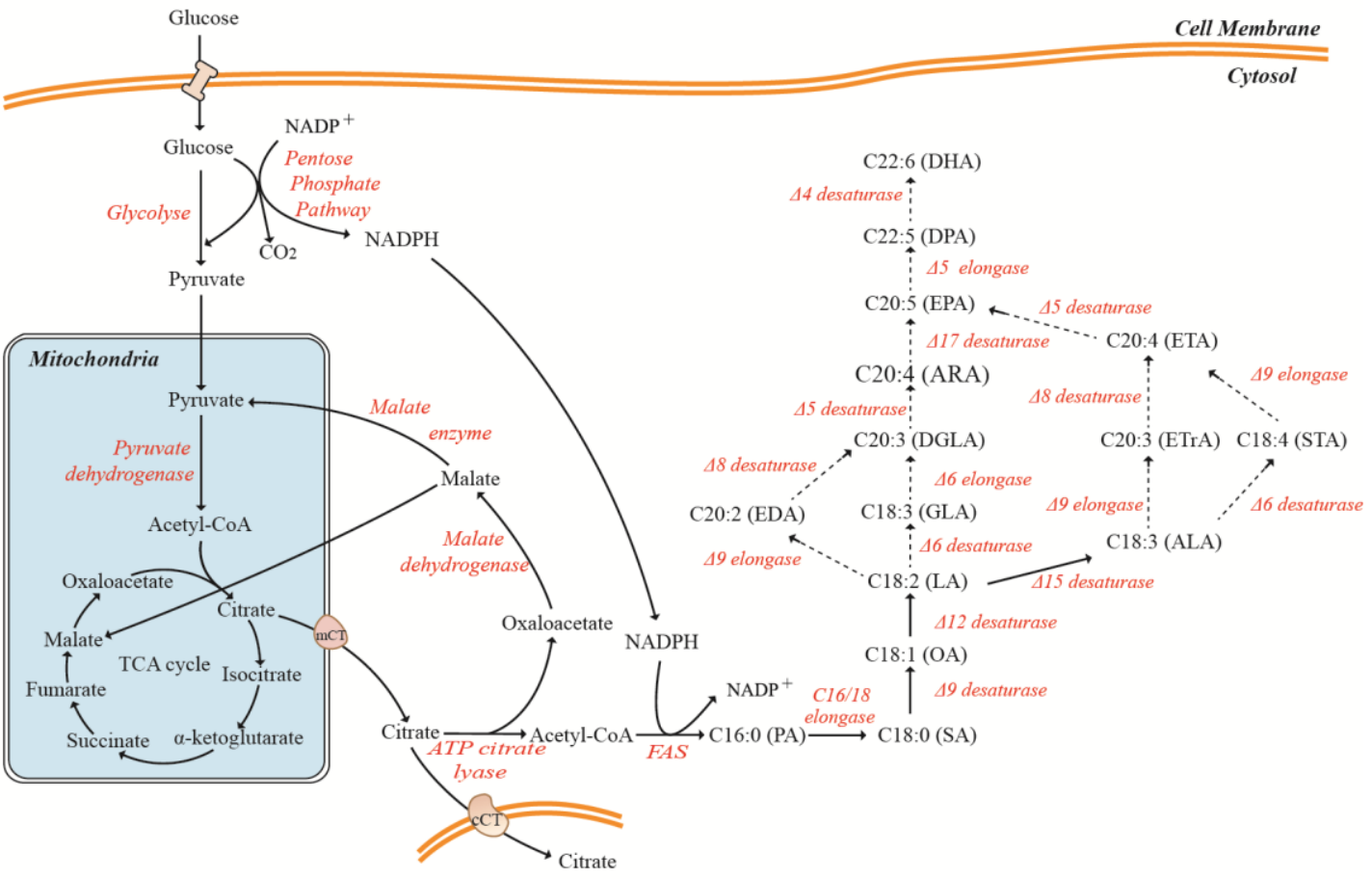

Fig. 1. 


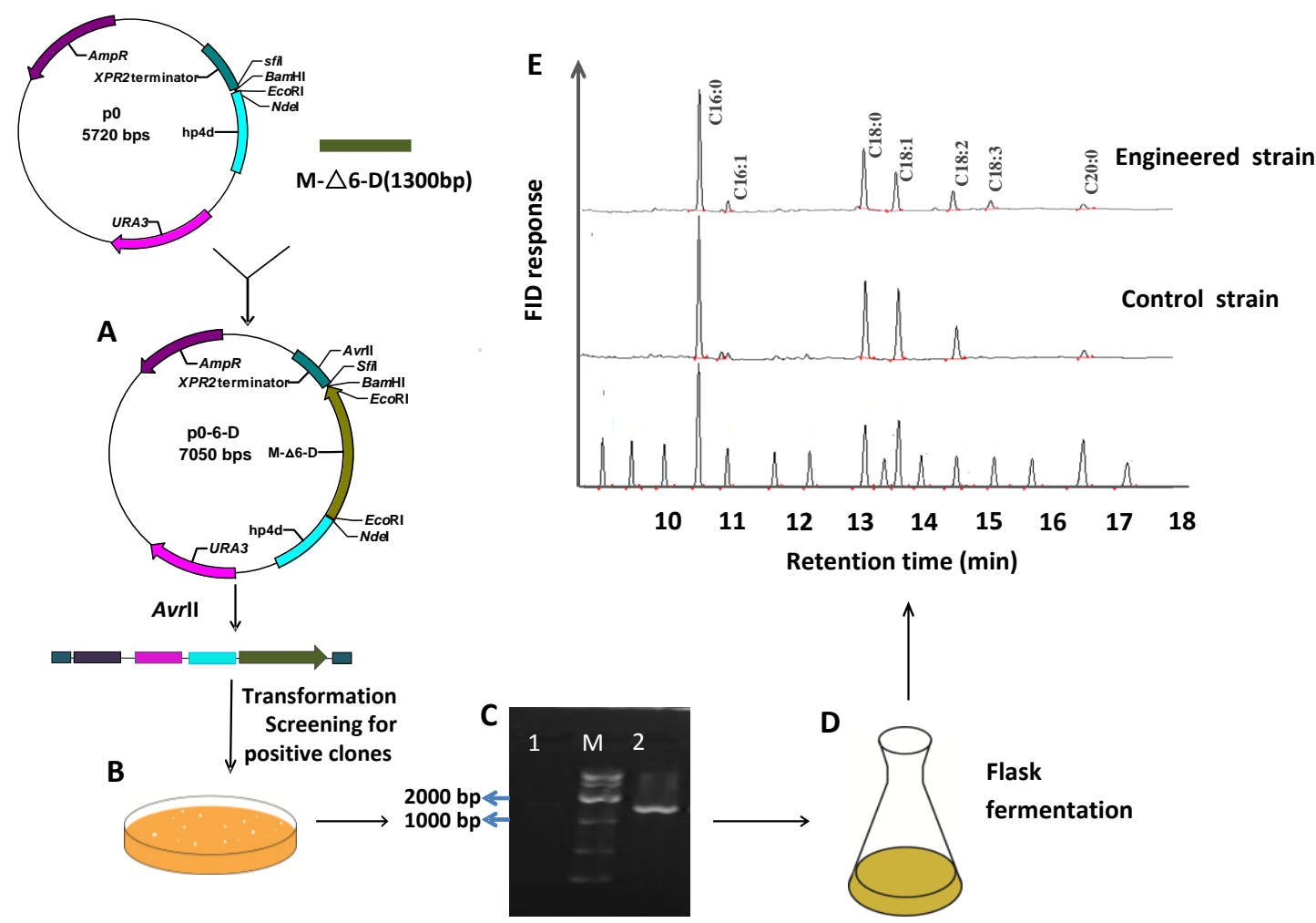

Fig. 2. 
1

2

3

4

5
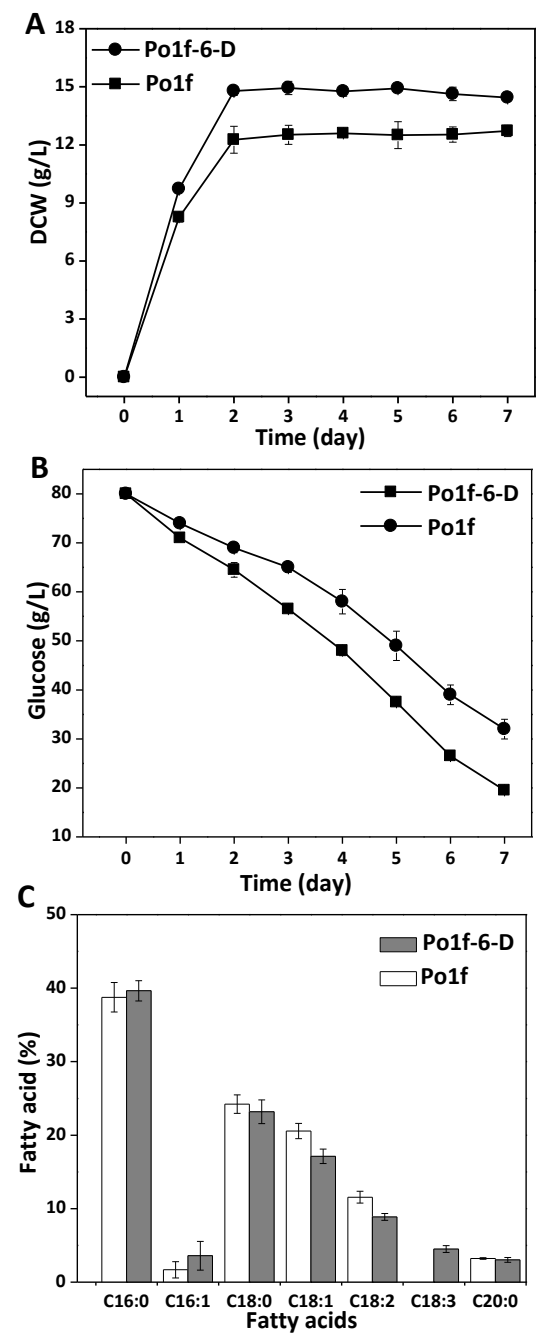

Fig. 3. 
1

2

3

4

5

6

7

8

10

11

12

13

14

15

16

17

18

19

20

21

22

23

24

25

26

27

28

29

30

31

32

33

34

35

36

37

38

39

40

41

42

43

44

45

46

47

48

49

50

51

52

53

54

55

56

57

58

59

60

61

62

63

64

65

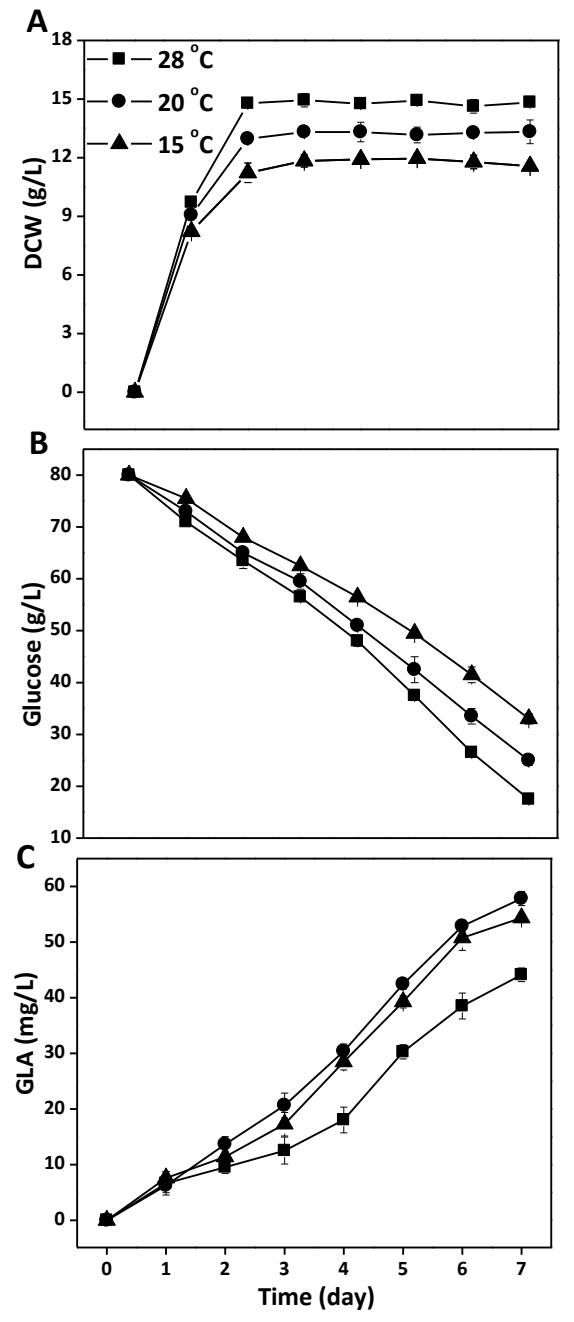

Fig. 4. 
A
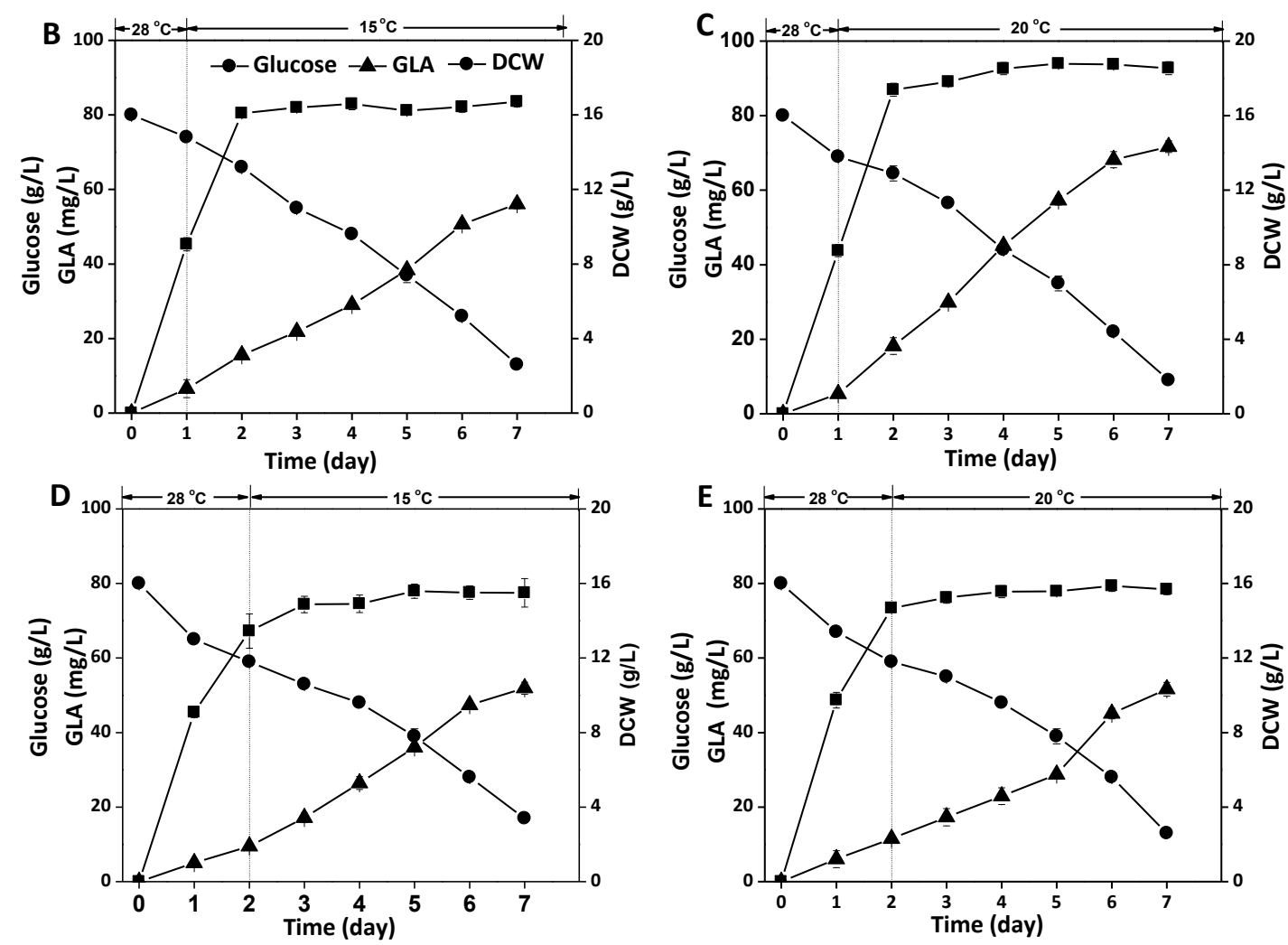

Fig. 5. 
Table 1 Comparison of data obtained at the end (day 7) of the engineered Yarrowia lipolytica strain Po1f-6-D fermentations at different temperatures.

\begin{tabular}{ccccc}
\hline Temperature $\left({ }^{\circ} \mathrm{C}\right)$ & DCW $(\mathrm{g} / \mathrm{L})$ & Lipid $(\mathrm{g} / \mathrm{L})$ & GLA(mg/L) & GLA/DCW(\%) \\
\hline 15 & 11.5 & 0.68 & 54.3 & 0.47 \\
20 & 13.3 & 0.83 & 57.4 & 0.43 \\
28 & 14.8 & 0.97 & 44.5 & 0.3 \\
$28(0-24 \mathrm{~h}) 15$ (after 24h) & 16.7 & 1.1 & 56.1 & 0.34 \\
$28(0-24 \mathrm{~h}) 20$ (after 24h) & 18.6 & 1.16 & 71.6 & 0.39 \\
$28(0-48 \mathrm{~h}) 15$ (after 48h) & 15.5 & 1.02 & 51.5 & 0.33 \\
$28(0-48 \mathrm{~h}) 20$ (after 48h) & 15.7 & 1.05 & 51.7 & 0.33 \\
Increase & $(+25.7 \%)$ & $(+19.6 \%)$ & $(+60.9 \%)$ & $/$ \\
\hline
\end{tabular}

${ }^{\mathrm{a}}$ Increase obtained by a shift to $20{ }^{\circ} \mathrm{C}$ after $24 \mathrm{~h}$, compared to the result obtained in standard cultivation conditions single temperature control experiment at $28{ }^{\circ} \mathrm{C}$. DCW: dry cell weight; GLA: $\gamma$-linolenic acid. 
Table 2 Fatty acid profiles of the engineered Yarrowia lipolytica strain Po1f-6-D under various temperature control strategies.

\begin{tabular}{cccccccc}
\hline \multirow{2}{*}{ Temperature $\left({ }^{\circ} \mathrm{C}\right)$} & \multicolumn{7}{c}{ Fatty acid composition (\%) } \\
\cline { 2 - 8 } & $\mathrm{C} 16: 0$ & $\mathrm{C} 16: 1$ & $\mathrm{C} 18: 0$ & $\mathrm{C} 18: 1$ & $\mathrm{C} 18: 2$ & $\mathrm{C} 18: 3$ & $\mathrm{C} 20: 0$ \\
\hline 15 & 28.47 & 5.01 & 17.82 & 21.51 & 15.11 & 7.99 & 4.07 \\
20 & 32.93 & 4.14 & 19.28 & 19.41 & 14.16 & 6.92 & 3.74 \\
28 & 39.64 & 3.60 & 23.19 & 17.12 & 8.87 & 4.55 & 3.03 \\
28 (0-24h) 20 (after 24h) & 30.03 & 5.37 & 19.41 & 20.49 & 14.85 & 6.17 & 2.40 \\
28 (0-48h) 20 (after 48h) & 33.41 & 4.06 & 20.32 & 19.98 & 13.58 & 4.92 & 3.65 \\
28 (0-24h) 15 (after 24h) & 32.70 & 2.73 & 20.99 & 22.12 & 11.15 & 5.10 & 5.20 \\
28 (0-48h) 15 (after 48h) & 34.99 & 3.60 & 20.53 & 21.02 & 13.47 & 5.09 & 2.29 \\
\hline
\end{tabular}

C16:0, palmitic acid; C16:1, palmitoleic acid; C18:0, stearic acid; C18:1, oleic acid; C18:2, linoleic acid; C18:3, $\gamma$ - linolenic acid; C20:0, arachidic acid. 
Table 3 Fatty acid profiles of the control strain Yarrowia lipolytica Po1f under various temperature control strategies. 


\section{Supporting information}

\section{Engineering Yarrowia lipolytica for efficient $\gamma$-linolenic acid production}

Mei-Li Sun ${ }^{1}$, Catherine Madzak ${ }^{2}$, Hu-Hu Liu ${ }^{1}$, Ping Song ${ }^{1,5}$, Lu-Jing Ren ${ }^{1,5}$, He Huang $^{3,4,5}$, Xiao-Jun Ji ${ }^{1,5 *}$

1. College of Biotechnology and Pharmaceutical Engineering, Nanjing Tech

University, No. 30 South Puzhu Road, Nanjing 211816, Peo`ple’s Republic of China

2.GMPA, AgroParisTech, INRA, Université Paris-Saclay, 78850, Thiverval-Grignon,

France

3. School of Pharmaceutical Sciences, Nanjing Tech University, No. 30 South Puzhu Road, Nanjing 211816, People's Republic of China

4. State Key Laboratory of Materials-Oriented Chemical Engineering, Nanjing Tech University, No. 5 Xinmofan Road, Nanjing 210009, People's Republic of China

5. Jiangsu National Synergetic Innovation Center for Advanced Materials (SICAM), No. 5 Xinmofan Road, Nanjing 210009, People's Republic of China

*Corresponding authors: Tel: +86 25 58139942; Fax: +86 2558139389

E-mail: xiaojunji@njtech.edu.cn (X.-J. Ji) 


\section{Supplementary Table 1}

The sequence of codon-optimized $\Delta 6$-fatty acid desaturase and $\Delta 6$-fatty acid desaturase

\begin{tabular}{|c|c|}
\hline Gene & quence \\
\hline $\begin{array}{l}\text { codon- } \\
\text { optimized } \\
\Delta 6 \text {-fatty } \\
\text { acid } \\
\text { desaturase }\end{array}$ & $\begin{array}{l}\text { ATGGCCGCCGCCCCCTCCGTCCGAACCTTCACCAGAGCCGAAATCCTGA } \\
\text { ACGCTGAGGCCCTGAACGAGGGCAAGAAGGACGCCGAGGCCCCTTCC } \\
\text { TCATGATCATCGACAACAAGGTGTACGACGTGCGAGAATTCGTGCCCG } \\
\text { ACCACCCTGGTGGTTCCGTGATTCTGACCCATGTCGGTAAGGACGGCAC } \\
\text { TGACGTCTTTGACACCTTCCACCCCGAGGCCGCTTGGGAAACCTGGCT } \\
\text { AATTTTTATGTTGGTGACATTCACGAGTCTGACCGAGACATCAAGAACG } \\
\text { ACGACTTTGCTGCCGAAGTCCGAAAGCTGCGAACCCTGTTTCAGTCCCT } \\
\text { GGGTTACTACGATTCTTCTAAGGCCTACTACGCCTTCAAGGTCTCCTTCA } \\
\text { ACCTGTGCATCTGGGGCCTGTCCACCTTCGTCGTCGCCAAGTGGGGTCA } \\
\text { AACCTCTACCCTCGCTAACGTTGTTTCTGCTGCTCTGCTGGGTCTGTTCT } \\
\text { GGCAGCAGTGCGGTTGGCTCGCCCACGATTTCCTGCACCATCAGGTCTT } \\
\text { CCAGGACCGATTCTGGGGCGACCTCTTTGGCGCTTTCCTCGGTGGCGTG } \\
\text { TGTCAGGGTTTCTCCTCCTCCTGGTGGAAGGACAAGCACAATACCCACC } \\
\text { ACGCCGCCCCCAACGTGCATGGTGAGGACCCCGACATCGACACTCACCC } \\
\text { CCTCCTGACCTGGTCCGAGCACGCTCTGGAGATGTTCTCCGATGTCCCC } \\
\text { GACGAGGAGCTCACCAGAATGTGGTCCAGATTCATGGTGCTGAACCAG } \\
\text { ACCTGGTTCTACTTCCCCATCCTGTCTTTCGCCCGACTCTCCTGGTGCCTC } \\
\text { CAGTCCATCCTCTTCGTCATGCCCAACGGTCAGGCTCATAAACCTTCTGG } \\
\text { TGCCCGAGTGCCTATTTCCCTGGTGGAACAGCTGTCCCTCGCCATGCAC } \\
\text { TGGACCTGGTATTTAGCCACCATGTTCCTGTTCGTGAAGGACCCTATCA } \\
\text { ACATGTTCGTGTACTTCCTGGTGTCTCAGGCCGTGTGTGGCAACCTGCT } \\
\text { GGCTCTGGTCTTTTCTCTGAACCACAACGGCATGCCCGTCATCTCCAAG } \\
\text { GAGGAGGCCGTCGACATGGATTTCTTCACCAAGCAGATCATCACCGGC } \\
\text { CGAGATGTCCACCCTGGCCTGTTCGCCAACTGGTTCACCGGCGGCCTGA } \\
\text { ACTACCAGATCGAGCATCACCTGTTCCCCTCCATGCCCCGACACAACTTC } \\
\text { TCCAAAATCCAGCCCGCCGTGGAGACCCTCTGTAAGAAGTACAACGTGC } \\
\text { GATACCACACTACCGGTATGATCGAGGGCACCGCCGAGGTCTTCTCCCG } \\
\text { ACTGAACGAGGTCTCTCGAGCCGCCTCTAAGATGGGTAAGGCTCAGTA } \\
\text { A }\end{array}$ \\
\hline $\begin{array}{l}\Delta 6 \text {-fatty } \\
\text { acid } \\
\text { desaturase }\end{array}$ & $\begin{array}{l}\text { ATGGCTGCTGCTCCCAGTGTGAGGACGTTTACTCGGGCCGAGATTCTGA } \\
\text { ATGCCGAGGCCCTGAATGAAGGCAAGAAGGATGCTGAGGCACCCTTCC } \\
\text { TGATGATCATCGACAACAAGGTGTACGATGTCCGCGAGTTTGTCCCTGA } \\
\text { TCATCCCGGTGGAAGTGTGATTCTCACGCACGTTGGCAAGGACGGCAC } \\
\text { TGACGTCTTTGACACTTTCCACCCCGAGGCTGCTTGGGAGACTCTTGCC } \\
\text { AACTTTTACGTTGGTGATATTCATGAGAGCGACCGCGACATCAAGAATG } \\
\text { ATGACTTTGCGGCCGAGGTTCGCAAGCTGCGTACCTTGTTCCAGTCTCTT } \\
\text { GGCTACTACGATTCTTCCAAGGCATACTACGCCTTCAAGGTCTCGTTCAA }\end{array}$ \\
\hline
\end{tabular}




\begin{tabular}{|l|l|}
\hline CCTCTGCATCTGGGGCCTGTCGACATTCGTTGTTGCCAAGTGGGGCCAG \\
ACCTCGACCCTCGCCAATGTAGTTTCGGCTGCGCTTTTGGGTCTCTTCTG \\
GCAGCAGTGCGGATGGTTGGCGCACGACTTTTTCATCACCAGGTCTTC \\
CAGGATCGTTTCTGGGGCGATCTTTTCGGTGCCTTCTTGGGAGGTGTCT \\
GCCAGGGTTTCTCATCCTCCTGGTGGAAGGACAAGCACAACACTCACCA \\
CGCCGCTCCCAACGTCCACGGTGAGGATCCCGACATTGACACTCACCCT \\
CTGTTGACCTGGAGTGAGCATGCTCTGGAGATGTTCTCGGATGTCCCTG \\
ACGAGGAGCTGACCCGCATGTGGTCGCGCTTCATGGTCCTCAACCAGA \\
CCTGGTTCTACTTCCCCATTCTCTCGTTTGCCCGTCTCTCCTGGTGCCTCC \\
AGTCTATTCTCTTTGTTATGCCTAACGGTCAGGCCCACAAGCCCTCGGGT \\
GCGCGTGTCCCATTTCCTTGGTCGAGCAGCTGTCTCTCGCTATGCACT \\
GGACCTGGTACCTCGCCACCATGTTCCTGTTCGTGAAGGATCCCATCAA \\
CATGTTTGTGTACTTTTTGGTATCGCAGGCTGTTTGCGGCAACTTGTTGG \\
CGCTTGTGTTCTCACTCAACCACAACGGTATGCCTGTGATTTCCAAGGA \\
GGAGGCAGTCGATATGGATTTCTTCACCAAGCAGATCATCACGGGTCGT \\
GATGTCCACCCTGGTCTGTTTGCCAACTGGTTCACAGGTGGATTGAACT \\
ACCAGATTGAGCATCACTTGTTCCCTTCGATGCCCCGCCACAACTTTTCA \\
AAGATCCAGCCTGCTGTCGAGACCCTGTGCAAAAAGTACAATGTCCGAT \\
ACCACACCACCGGCATGATTGAGGGAACTGCAGAGGTCTTAGCCGTCT \\
GAACGAGGTCTCCAGGGCTGCCTCCAAGATGGGCAAGGACAGTAA \\
\hline
\end{tabular}

